\title{
Qualität als Thema organisationaler Selbstbeschreibungen: Marketing und Verbraucher*innenschutz?
}

\author{
Eine Dokumentenanalyse
}

\section{Stefan Klusemann (D) Marlena Kilinc $\cdot$ Dieter Nittel}

Eingegangen: 9. Juni 2021 / Überarbeitet: 9. November 2021 / Angenommen: 9. November 2021 / Online publiziert: 10. Januar 2022

(C) Der/die Autor(en) 2021

Zusammenfassung Als die Qualitätsdiskussion im Erziehungs- und Bildungswesen an Intensität gewann, spielte auch der ursprünglich im Wirtschaftssystem verortete Verbraucher*innenschutz eine Rolle. Wir gehen der Frage nach, in welcher Relation dieses ursprüngliche Motiv und ein damit korrespondierendes Marketing zur Form und zum Inhalt organisationaler Selbstbeschreibungen (OSB) von pädagogischen Organisationen im System des lebenslangen Lernens steht. Auf der Basis einer Dokumentenanalyse von 80 OSB von Einrichtungen in vier Segmenten (Elementarbereich; Sekundarstufe II; Weiterbildung; Sozialarbeit/Sozialpädagogik) zeigen wir, dass der Verbraucher*innenschutz eine zentrale Rolle spielt: das Marketing, in das der Qualitätsbegriff eingebunden ist, wird nicht einseitig durch anpreisende, werbeaffine Sprachmittel dominiert; es übernimmt vielmehr Informationsfunktionen. Zwar erfüllen die Qualitätsmanagementsysteme nicht die ursprünglich erwartete Orientierungsfunktion; das aus der Privatwirtschaft stammende Konzept des Verbraucher*innenschutzes ist bereichsübergreifend jedoch als emphatische Klient*innenorientierung angeeignet worden sowie in Form eines Selbstverständnis der Einrichtungen als lernende Organisationen, einschließlich der Überprüfung und Bewertung der eigenen Arbeit durch die Adressat*innen. Diese bereichsübergreifende Homologie konstituiert eine systemweite Definition guter pädagogischer Praxis,

\footnotetext{
Die Analyse ist Teil des von der Deutschen Forschungsgemeinschaft geförderten QUEB-Projekts (Qualitätsentwicklungen im Erziehungs- und Bildungswesen: Varianten eines reflexiven Mechanismus). Ziel ist eine segmentübergreifende, vergleichende Rekonstruktion - unter organisations- und professionstheoretischen Gesichtspunkten - wie pädagogische Organisationen sich das Qualitätsthema aneignen und welche Erfahrungen sie mit Qualitätsentwicklung machen. Die Analyse beruht auf organisationalen Selbstbeschreibungen (OSB), Expert*innen-Interviews mit Leitungen sowie Gruppendiskussionen mit pädagogisch Tätigen in vier Segmenten.
}

Dr. Stefan Klusemann $(\varangle) \cdot$ Marlena Kilinc $\cdot$ Prof. Dr. Dieter Nittel

Lehrgebiet Empirische Bildungsforschung, FernUniversität in Hagen, Universitätsstraße 33,

Gebäude 1, 58097 Hagen, Deutschland

E-Mail: stefan.klusemann@fernuni-hagen.de 
die den Aspekt des Verbraucher*innenschutzes systemimmanent adaptiert hat. Damit verdeutlichen die Daten Systembildungstendenzen hin zu einem gemeinsamen System des lebenslangen Lernens. Von einer bloßen Ökonomisierung des Systems durch die Adaptierung des Qualitätskonzepts kann nicht die Rede sein. Den Befunden kommt in mehreren Punkten Praxisrelevanz zu: Einrichtungen könnten den Aspekt des Verbraucher*innenschutzes expliziter aufgreifen; dieser Aspekt von Ökonomisierung kann mit positivem Selbstverständnis hervorgehoben werden und die Anerkennung des Systems stärken. Zudem sollten Einrichtungen die Textsorte der OSB im Internet in ihrer Bedeutung noch ernster nehmen. Einzelne Einrichtungen sehen darin ein notwendiges Übel; OSB sind jedoch für die Einrichtungen und das System objektiv relevant.

Schlüsselwörter Qualität · Qualitätsmanagement-Systeme · Organisationale Selbstbeschreibungen · Verbraucher*innenschutz $\cdot$ Emphatische Klient*innenorientierung $\cdot$ Systembildungstendenzen

\section{Quality as a topic in organizational self-descriptions: marketing and consumer protection?}

A document analysis

Abstract When quality-management-systems became a subject of discussion within the educational system, the economic concept of consumer-protection was a central goal. It had been associated-in the economic system-with quality-managementsystems from their start. In this paper, we address the question: what is the relation between the original motive of consumer-protection (and its corresponding aspect of marketing) and the form and content by which organizations within the educational system address the quality topoi in their organizational-self-descriptions (OSB). We do so by analyzing 80 organizational-self-descriptions from four segments (kindergarten; high schools; andragogy; social work). We find that the concept of consumer protection plays a crucial role: the marketing, in which the quality topoi is embedded, is not one-sidedly dominated by an aggressive, advertising language but serves a function of information. And the quality-topoi itself serves a function of consumer protection in a modified form: the economic concept of quality-management-systems does not serve the original goal of an enhanced orientation for (potential) clients. But the organizations re-formulated — as in a hidden curriculum - the original motive of consumer-protection: System-wide, the concept has turned into an emphatic clientorientation as well as a self-understanding as learning organizations (i.e., a continual advancement of professionality as well as a continual review and assessment of the work-including by their clients). This homology—across segments-constitutes a systemwide definition of good pedagogical practice and transforms the concept of consumer-protection. On a theoretical level, the homology supports the thesis of system-building tendencies. The data does not support the thesis of a simple economization of the system by way of adopting the quality topoi. Instead, we find a highly constructive process of appropriation. Our findings also have some implications for educational practice: pedagogical organizations should explicitly take up the concept of consumer protection; it represents a positive aspect of economization 
that can be taken up affirmatively and which might in turn foster the profession's recognition. Furthermore, organizations should take organizational self-descriptions in the internet more seriously. Some regard it primarily as a necessary evil; however, organizational self-descriptions are of objective relevance for the individual organizations as well as the system as a whole.

Keywords Quality · Quality management systems · Organizational selfdescriptions · Consumer protection · Emphatic clientorientation · System-buildung tendencies

\section{Ausgangssituation: Qualität als ubiquitäres Element des pädagogisch organisierten Systems des lebenslangen Lernens}

Die LOEB-Studie (,Die Resonanz des lebenslangen Lernens in Organisationen des Erziehungs- und Bildungswesens“, Nittel und Tippelt 2018, 2019) hat aufzeigen können, dass in allen Segmenten des pädagogisch organisierten Systems des lebenslangen Lernens, ${ }^{1}$ d.h. in der Elementarpädagogik, im Primarbereich, in der Sekundarstufe I und II, in der beruflichen Bildung, in der Sozialarbeit/Sozialpädagogik, in der Erwachsenenbildung/Weiterbildung und im Hochschulbereich, das Thema Qualität allgegenwärtig ist. ${ }^{2}$ Mit der Ubiquität des Qualitätsthemas korrespondiert jedoch nicht nur die Frage, ob die Mehrzahl pädagogischer Organisationen ${ }^{3}$ aufwendige Verfahren des Qualitätsmanagements (QM) - wie etwa die ISO-Normenreihe oder EFQM - umsetzt oder Qualitätsbeauftragte benennt. Diese Kategorie zielt vielmehr auf ein tieferliegendes, strukturelles Phänomen ab, das sowohl das Wissen als auch das Handeln der Akteur*innen tangiert: Vertreter*innen von Kindergärten, Schulen, Einrichtungen der Erwachsenenbildung, der Sozialarbeit und von Hochschulen müssen sich - so zeigt die Triangulation der Daten der LOEB-Studie - mit dem Qualitätsphänomen auf der Ebene ihres Berufswissens argumentativ auseinandersetzen und diesbezüglich eine Position beziehen: Sie können sich nicht nicht-

\footnotetext{
1 Zur Begründung und Definition der handlungs- und nicht kommunikationstheoretisch fundierten Kategorie des pädagogisch organisierten Systems des lebenslangen Lernens verweisen wir auf eine Vielzahl bereits publizierter Beiträge (Nittel 2017; Nittel et al. 2014; Nittel und Tippelt 2018, 2019).

2 Dieser Befund ist sehr belastbar, weil er auf der Analyse von organisationalen Selbstbeschreibungen, Expert*innen-Interviews mit Einrichtungsleiter*innen und Gruppendiskussionen mit Vertretern*innen des operativ tätigen Personals (Erzieher*innen, Kursleitungen etc.) aus vier verschiedenen Regionen Deutschlands beruht.

3 Wir legen den Organisationsbegriff der LOEB-Studie zugrunde (Nittel und Tippelt 2019; Wahl et al. 2022). Dieser ist angelehnt an Kuper und Thiel (2018) sowie die Theorie des Neoinstitutionalismus. Organisationen sind demnach durch die Dimensionen ,Zweckorientierung, [...] Arbeitsteilung, formale Mitgliedschaft oder Mitgliedsrollen und eine dauerhafte Abgrenzung zur Umwelt“ (Kuper \& Thiel 2018, S. 596) charakterisiert. In Adaptierung des Neoinstitutionalismus betonen wir zudem die Interaktion von Organisationen mit ihren Umwelten und die daraus entstehenden (internen) Aushandlungsprozesse, ,die zur Anpassung der Organisationkultur führen können [...] und ihre Flexibilität gewährleisten. Bei diesen Aushandlungsprozessen spielen [...] (OSB) eine zentrale Rolle, da in ihnen identitätsstiftende Elemente der jeweiligen Organisationskultur fixierbar werden. Auch müssen sie die Perspektive der Organisationsmitglieder mit den Erwartungen der Außenwelt [...] abgleichen.“ (Wahl et al. 2022, o.S.).
} 
kommunikativ zu diesem Thema verhalten. Zugleich stehen Einrichtungen unter dem Zugzwang, diesbezüglich eine Praxis zu etablieren, die über bloße Absichtserklärungen hinausgeht. Dementsprechend wurde die Frage danach, $o b$ Qualität im Zentrum der Aufmerksamkeit der Einrichtungen steht, durch die Frage nach dem wie ihrer Umsetzung ersetzt. ${ }^{4}$

\section{Fragestellung und Darstellungsüberblick}

Im vorliegenden Beitrag verbinden wir die Beobachtung der Allgegenwart des Qualitätsphänomens mit der Betrachtung eines anderen zentralen Themas: dem Verbraucher*innenschutz. Der Verbraucher*innenschutz war eines der zentralen Motive zur Entwicklung von Qualitätsmanagementsystemen (QMS). ${ }^{5}$ Gründungsidee der im Wirtschaftssystem entwickelten ISO-Normenreihe ${ }^{6}$ war etwa, Transparenz für Kund*innen zu schaffen, ihnen mittels Komplexitätsreduzierung Orientierung zu liefern und die Leistungsfähigkeit der Anbieter*innen zu verbriefen (Deutsche Gesellschaft zur Zertifizierung von Managementsystemen, o.J.).

Dieser im Wirtschaftssystem zentrale Gedanke des Verbraucher*innenschutzes spielte auch eine wichtige Rolle, als die Qualitätsdiskussion vor 30 Jahren im pädagogisch organisierten Systems des lebenslangen Lernens an Intensität gewann. ${ }^{7}$ Die Beglaubigung von Qualität und ihre hiermit einhergehende öffentlichkeitswirksame Präsentation könnten - so eine bildungspolitische Annahme - die Orientierung der Adressat*innen erleichtern. Im Qualitätsdiskurs der Weiterbildung waren der Verbraucher*innenschutz (Weiterbildung Hessen o.J.; Wuppertaler Kreis e. V. 1996, S. 3) - auch im Zusammenhang mit Bildungstests (Hartz und Meisel 2011) - und „die Schaffung von Vergleichbarkeit“ und der Teilnehmer*innenschutz von zentraler Relevanz (ebd., S. 95). Auch im Elementarbereich war es ein wichtiges Ziel, „dass [...] Eltern in der Auswahl von Einrichtungen eine Orientierungsmöglichkeit

\footnotetext{
4 In zwei Sammelpublikationen der Zeitschrift für Pädagogik, die beide den Anspruch einer Zwischenbilanz erheben, wird die Geschichte der Qualitätsdiskussion in den einzelnen Segmenten in ihren Grundzügen nachgezeichnet (Helmke et al. 2000; Klieme und Tippelt 2008).

5 In Anlehnung an Merchel verwenden wir eine eher weit gefasste Begriffsbestimmung von QMS und subsumieren hierunter diejenigen als solche etikettierten Systeme von Verfahren, welche die kontinuierliche und systematische Bewertung, Erzeugung und Beeinflussung der Qualität sozialer Dienstleistungen verfolgen (Merchel 2013, S. 15). Dabei ist zentral, dass ein QMS als System mit einem Handlungsfokus angelegt ist, den alle Maßnahmen teilen. Einzelne Maßnahmen sind zu einem logischen Ganzen verbunden. Dementsprechend fallen hierunter nicht nur die aus dem Wirtschaftssystem stammenden Konzepte, wie die ISO (International Organization for Standardization) Norm 9001 oder EFQM (European Foundation for Quality Management), sondern auch QMS, die segmentspezifisch von Trägern, Verbänden oder Organisationen entwickelt wurden (wie bspw. LQW (Lernerorientierte Qualitätstestierung in der Weiterbildung); das Gütesiegel des Verbandes katholischer Tageseinrichtungen für Kinder (KTK) oder das QMS des ,Gütesiegelverbundes NRW`). Zudem binden wir den Begriff nicht ausschließlich an externe Zertifizierungen und berücksichtigen auch Verfahren, die auf Selbstevaluationen beruhen.

6 Die „International Organization for Standardization“ (ISO) wurde im Jahr 1946 mit dem Ziel gegründet, international vergleichbare Standards (für die Industrie) zu entwickeln (vgl. International Organization for Standardization, o.J.).

7 Nichtsdestotrotz wurde in der bildungswissenschaftlichen Literatur zu QMS und Qualitätsentwicklungen der Aspekt des Verbraucher*innenschutzes, wenn überhaupt, nur implizit aufgenommen.
} 
erhalten“ (Dittrich o.J., o.S.; Spieß und Tietze 2002). Die Einführung des Deutschen Kindergarten Gütesiegels wurde beispielweise damit begründet, „dass Eltern für die Ausübung ihres ordnungspolitisch gewollten Wunsch- und Wahlrechts (Kinder- und Jugendhilfe-Gesetz KJHG, § 5) einer Qualitätsinformation bei den zur Auswahl stehenden Einrichtungen bedürfen“ (Tietze 2008, S. 29). Auch in der Sozialarbeit/ Sozialpädagogik äußerten Befürworter*innen die Erwartung, dass QMS eine nutzer*innenfreundliche Komplexitätsreduktion leisten und insofern „Orientierung bei der Wahl der Angebote“ liefern könnten (Merchel 2004, S. 49). QMS würden „die Unübersichtlichkeit bei den Angeboten und die daraus resultierende Unsicherheit bei Adressaten“ reduzieren (ebd.; Brückers 1998).

Es sollte deutlich geworden sein, dass Qualität ursprünglich auch mit Erwartungen an Transparenz, Vergleichbarkeit und Orientierung verbunden war. Da der Qualitätstopos somit per se eine Verbindung zur Umwelt der Organisationen aufweist, ist er zur Durchsetzung dieser Ansprüche auf Öffentlichkeitsarbeit oder - anders ausgedrückt - auf ein gewisses Marketing angewiesen. Verbraucher*innenschutz setzt Kommunikation voraus und das kann nicht ohne Marketing erfolgen. ${ }^{8}$ Eine Doppelfunktion von QMS als Instrument des Verbraucher*innenschutzes und des Marketings wurde bereits in den 1990er-Jahren unterstrichen. Man ging mit Bezug auf das Qualitätsthema von einer Parallelität aus (Wuppertaler Kreis 1996).

Vor diesem Hintergrund gehen wir in diesem Artikel der Frage nach, in welcher Relation das ursprüngliche Motiv des Verbraucher*innenschutzes und eine hiermit (d.h. mit Orientierung und Informierung) korrespondierende Marketingfunktion sich zur Form und zum Inhalt jener Textgattung verhalten, auf welche die Zielgruppen von Erziehungs- und Bildungsangeboten (oder deren gesetzliche Vertreter*innen) zunehmend zurückgreifen, um sich über die Qualität einer Organisation zu informieren: die organisationalen Selbstbeschreibungen (OSB) der Einrichtungen (oder ihrer Träger) im Internet. ${ }^{9}$

Im vorliegenden Artikel präsentieren wir zunächst OSB als unsere Datengrundlage und stellen das methodische Vorgehen unserer Dokumentenanalyse vor (Abschn. 3). Anschließend präsentieren wir die Ergebnisse unserer Analyse (Abschn. 4) und widmen uns hierbei zunächst der Frage nach dem ,Wie" des Marketings im Zusammenhang mit dem Qualitätsbegriff (Abschn. 4.1) und erörtern anschließend, inwiefern der Verbraucher*innenschutz Bestandteil des Qualitätstopos in den OSB ist (Abschn. 4.2 und 4.3). Nach Beantwortung der Forschungsfrage

\footnotetext{
8 Wir betrachten den Begriff des Marketings als wertneutral. Es ist die Kommunikationsform mit der Umwelt, die Organisationen nutzen, die der Knappheitsmaxime gerecht werden müssen. Wir legen die Definition von Grönross zugrunde: „Marketing is a customer focus that permeates organizational functions and processes and is geared towards making promises through value proposition" (Grönroos 2006, S. 407).

9 Für die Informationsbeschaffung über eine Einrichtung spielt heute - so unsere Einschätzung auf der Basis unmittelbarer Erfahrungen aus dem Feld im Rahmen des QUEB-Projekts - das Internet eine zentrale Rolle. Aktuelle Studien zur Informationsbeschaffung und zur Nutzung der OSB durch Adressat*innen liegen nach unserem Kenntnisstand nicht vor. Hier besteht weiterer Forschungsbedarf. Durch 53 Expert*innen-Interviews mit Leitungen wissen wir jedoch, dass in der Wahrnehmung der Einrichtungen die Adressat*innen sich vor allem im Internet über die Organisation informieren. Vor diesem Hintergrund wird die strategisch wichtige Rolle von OSB erkennbar, die im Internet für alle Bürger*innen frei zugänglich sind. Trotz ihrer augenfälligen Relevanz im System werden OSB jedoch bisher von der empirischen Bildungsforschung kaum beachtet.
} 
diskutieren wir in Abschn. 4.4 eine zentrale Funktion der in den OSB identifizierten Formen von Verbraucher*innenschutz: die Legitimationsfunkton (Abschn. 4.4). Abschließend skizzieren wir Implikationen für die Praxis und ziehen weitergehende Schlussfolgerungen aus unseren Erkenntnissen für das pädagogisch organisierte System des lebenslangen Lernens (Abschn. 5).

\section{Organisationale Selbstbeschreibungen als Forschungsgegenstand}

In der LOEB-Untersuchung sind erstmalig in der Geschichte der empirischen Bildungsforschung OSB im größeren Maßstab als Datenmaterial genutzt worden. ${ }^{10} \mathrm{Un}$ ter OSB verstehen wir eine kommunikative Gattung offizieller Texte. Mit ihrer Hilfe bringen pädagogische Organisationen zum Ausdruck, dass sich sowohl die Leitung als auch die Mitarbeiter*innen mit der eigenen Organisation identifizieren, diese soziale Einheit ein kommunikatives Gedächtnis besitzt und gegenüber der sozialen Umwelt über ein abgrenzbares Profil verfügt. In OSB finden sich unterschiedliche Angaben, etwa der organisationale Auftrag oder orientierungsmächtige Maximen.

Sowohl die Analysen der OSB im Rahmen des LOEB-Projekts als auch in der QUEB-Studie beschäftigen sich somit mit dem von den Einrichtungen formulierten Selbstbild. Wir machen insofern auch nur über die diskursive Umsetzung des Qualitätsthemas in den OSB Aussagen und nicht über die Qualität der Einrichtungen.

Während sich die Analyse des LOEB-Projekts auf die impliziten Indikatoren des Qualitätsthemas in OSB richtete, untersucht die QUEB-Studie, wie Organisationen den Qualitätsbegriff explizit verwenden. Ein Ziel ist die Bestimmung der spezifischen inhaltlichen Bezüge und der formalen Merkmale der Qualitätsthematisierungen. Die im Rahmen dieses Artikels zugrundeliegenden Fragen bilden einen ersten Schritt dieser Dokumentenanalyse, die auf 80 OSB von Einrichtungen aus vier ausgewählten Segmenten (Elementarbereich, Sekundarstufe II, Sozialarbeit/ Sozialpädagogik und Weiterbildung) in vier Bundesländern (Bayern, Brandenburg, Hamburg und Nordrhein-Westfalen) beruht. ${ }^{11}$

Für die Analyse wurden alle Absätze der OSB herangezogen, in denen der Qualitätsbegriff explizit verwendet wird. Diese Ausschnitte fungierten als Analyseeinheiten. Im ersten Teil der Untersuchung fokussierten wir dabei auf das ,Wie' des Marketings. Dabei wurden zunächst im Rahmen einer Auswertung mit der Grounded Theory Stilmittel eines anpreisenden Marketings in den OSB identifiziert. Anschließend wurden die Analyseeinheiten einer deduktiven Inhaltsanalyse (Mayring

\footnotetext{
10 Auf der Basis einer Totalerhebung von 417 Dokumenten in einer städtischen und einer ländlichen Region wurde ein Theoretical Sample von 52 kontrastierenden OSB ausgewählt. Die Studie kam zu dem Befund, dass die Organisationen mit der Einführung von Qualitätsentwicklungsmaßnahmen eine grundlegende Idee des lebenslangen Lernens auf sich selbst applizieren: die eigene Innovationsfähigkeit im Medium des Lernens unter Beweis zu stellen.

11 Die Auswahl der 80 OSB erfolgte im Kontext des qualitativ-interpretativen Kontextes des QUEB-Projekts. Die Fallauswahl erfolgte nicht zufallsgesteuert und eine statistische Repräsentativität war nicht ihr Ziel. Maßgebend war das Prinzip der Berücksichtigung größtmöglicher Kontraste, um die Heterogenität der Segmente bei der Theoriebildung einbeziehen zu können. Ausgewählt wurden dabei nur Organisationen, die den Qualitätsbegriff explizit in ihren OSB verwenden.
} 
2015) unterzogen. Dabei fungierten (a) die identifizierten Stilmittel eines anpreisenden Marketings und (b) die Kommunikationsschemata der Sachverhaltsdarstellung (Dellori 2016; Kallmeyer und Schütze 1977; Schütze 1978) als Kategorien. Darauf aufbauend wurden Aussagen dazu getroffen, in welchen OSB die Verwendung des Qualitätsbegriffs von anpreisenden Werbe-Stilmitteln dominiert wird. Hier legen wir einfache Häufigkeitszählungen zugrunde: wir sprechen von einem dominant anpreisenden Stil einer OSB, wenn eine - im Segmentvergleich - deutliche Häufung anpreisender Stilmittel zu finden ist oder wenn die identifizierten Segmente mit anpreisenden Stilmitteln diejenigen deutlich überwiegen, denen auf der Basis der Kommunikationsschemata eine Informationsfunktion zugeordnet werden kann. ${ }^{12} \mathrm{Im}$ zweiten Teil der Auswertung wurden (a) verwendete QMS identifiziert und (b) eine inhaltlich strukturierende qualitative Inhaltsanalyse nach Kuckartz (2018) vorgenommen, kombiniert mit einer Frequenzanalyse zur Herausarbeitung der häufigsten inhaltlichen Bezüge, mit denen der Qualitätsbegriff in den OSB genannt wird (Bessler 1972, S. 76-78). Damit werden Häufigkeitsaussagen dazu möglich, wie der Qualitätsbegriff von den pädagogischen Organisationen adaptiert wurde, und in welchem Verhältnis dies zur Grundidee des Verbraucher*innenschutzes steht. ${ }^{13}$

\section{Ergebnisteil: Erste Tendenzen - erste Befunde}

In diesem Kapitel beantworten wir die Forschungsfrage. Dazu präsentieren wir zunächst in Abschn. 4.1 Befunde dazu, inwiefern das Qualitätsthema in den OSB einseitig von einem offensiven, anpreisenden Marketing mit werbeaffinen Sprachspielen dominiert wird. Anschließend betrachten wir in Abschn. 4.2, inwiefern den in den OSB aufgeführten QMS eine Orientierungsfunktion im Sinne des Verbraucher*innenschutzes zukommt. Eine solche Orientierungsfunktion muss auf der Basis der Daten verneint werden. Abschn. 4.3. zeigt jedoch, dass der Aspekt des Verbraucher*innenschutzes systemweit sehr wohl aufgegriffen und systemimmanent adaptiert wurde. Abschn. 4.4 ergänzt, dass die in den OSB enthaltenen Aspekte des Verbraucher*innenschutzes eine Legitimationsfunktion erfüllen.

\subsection{Der Qualitätsbegriff und Marketing mit anpreisenden Stilmitteln}

Die Textgattung der OSB stellt ein Artefakt der Öffentlichkeitsarbeit pädagogischer Organisationen dar. Ihnen ist per se auch eine Marketingkomponente inkorporiert; der Verwendungs- und Anwendungskontext evoziert bereits Marketing. In unseren Analysen konnten wir formale Stilmittel identifizieren, die man einem Marketing

\footnotetext{
${ }^{12}$ Um über Häufigkeitsauszählungen hinausgehende Aussagen machen zu können ist weitere Forschung erforderlich, beispielsweise durch die Adaptierung einer Contingency Analyse (Bessler 1972, S. 78). Damit würde überprüfbar, inwiefern es sich bei der Verwendung von anpreisenden Sprachspielen im Zusammenhang mit dem Qualitätsbegriff um ein mehr als zufälliges gemeinsames Auftreten handelt (ebd., S. 79).

${ }^{13}$ Die vorliegende Analyse stützt sich auf Häufigkeiten in semantischen Bezügen. Weitergehende Aussagen dazu, welches Qualitätsverständnis die OSB aufweisen, welche Funktionen sie dem Qualitätsthema zuordnen und wie sie den Prozess von Qualitätsentwickelung darstellen, erfolgen im Rahmen separater Publikationen.
} 
zuordnen kann, dem eine primäre Werbefunktion im Sinne des Anpreisens einer Dienstleistung inhärent ist. Anhand des nachfolgenden Beispiels können verallgemeinerungsfähige Aspekte einer solchen anpreisenden, offensiven Marketingstrategie dargestellt werden:

\section{QUALITÄT}

WIR SIND EIN FAMILIENGEFÜHRTES UNTERNEHMEN.

Wir haben uns den allerhöchsten Ansprüchen in der Kinderbetreuung verschrieben. Unsere Motivation liegt in der Leidenschaft und der Freude an der frühkindlichen Bildung. Ein selbstvertrauendes, starkes und glückliches Kind ist die wunderbarste Form der Bestätigung.

Der hier dokumentierte Auszug stammt von einem privatwirtschaftlichen Unternehmen - einem Träger, der Einrichtungen der Elementarpädagogik unterhält. Auf der Ebene der Beziehungskonstitution geht es darum, über im Wirtschaftsleben bekannte, aber in pädagogischen Handlungszusammenhängen eher überraschende Assoziationen (,Wir sind ein familiengeführtes Unternehmen“) Vertrauen zu schaffen. Durch formale Aspekte wie Großbuchstaben oder andere besonders hervorstechende Layoutmerkmale, wie eine auffällige Farbwahl, findet eine Aufmerksamkeitsfokussierung statt: Den Leser*innen wird die Zentralität eines Identitätsmarkierers (Goffman 1963, 1967) vermittelt, der im Hinblick auf die Typik der Organisation die ja eine familienbegleitende Betreuung anbietet - nicht ganz unwesentlich sein dürfte. Durch Superlative (,allerhöchsten Ansprüchen“) und die auch in anderen Beschreibungen zu findende Steigerungsrhetorik eines immer-besser-Werdens wird explizit überdurchschnittliche Leistungsbereitschaft und -vermögen und implizit ein Wettbewerbsvorsprung gegenüber der Konkurrenz signalisiert. Zudem wird durch den gezielten Bruch von Regeln kommunikativen Handels (Behauptungen in Werbeformaten werden häufig weder begründet noch belegt, wodurch die Zugzwänge der Argumentation situativ suspendiert werden) ein Akt der Selbstermächtigung vollzogen, der Autorität und Selbstevidenz ausdrücken soll: Die Organisation hat es nicht nötig, Aussagen im Zusammenhang mit Qualitätsbehauptungen zu belegen. In den nachfolgenden Passagen dieser OSB können wir zudem Hinweise auf aktuelle Themen (gesunde Ernährung und Umweltschutz) identifizieren. Hierdurch soll Aktualität signalisiert und die eigene Einrichtung am Puls der Zeit platziert werden. Der hier angesprochene Typ von OSB hat im Kern die Funktion, über Selbstattribuierungen so etwas wie eine Marke zu etablieren.

In anderen OSB können weitere Stilmittel einer anpreisenden Werbesprache ausgemacht werden. Beispielsweise ein Fokus auf Selbstoptimierung oder holistische und listenartige Darstellungen. Mit möglichst wenig Text sollen möglichst viele Akteur*innen genannt bzw. adressiert oder Qualitätsdimensionen aufgezählt werden. Bildungsbereichsübergreifend sind die beiden Aspekte der Selbstoptimierung und der allumfassenden Orientierung (neben der Steigerungsrhetorik und sloganhaften Sätzen) die am häufigsten vorkommenden werbeaffinen Stilmittel in den von uns untersuchten OSB. Im nachfolgenden Auszug werden sie beispielhaft illustriert:

Unsere Qualitätsstandards ,,konkretisieren sich in der Kontinuität und arbeitsmarktpolitischen Aktualität unseres Angebotes, der hohen fachlichen, päda- 
gogischen und sozialen Kompetenz unserer Mitarbeiter*innen, unserer guten räumlichen und technisch-medialen Ausstattung, unseren erwachsenengerechten Lehr- und Lernmaterialien, der Kundenorientierung des gesamten Unternehmens. Qualität ist für uns keine feststehende Größe. Sie ergibt sich vielmehr aus einem ständigen Optimierungsprozess, der sich nicht mit den jeweils erreichten Standards zufrieden gibt.“

Unsere deduktive Inhalts- und Häufigkeitsanalyse zeigt, dass anpreisende Stilmittel jedoch nur in wenigen OSB das Qualitätsthema dominieren. Verortet sind sie bei privatwirtschaftlichen Organisationen. Im Elementarbereich und der Sozialarbeit/ Sozialpädagogik kann nur bei $20 \%$ bzw. 10\% von den jeweils 20 analysierten OSB beobachtet werden, dass die Thematisierung des Qualitätsbegriffs deutlich von einer anpreisenden Werbesprache geprägt ist. ${ }^{14}$ Für den am stärksten etablierten und autonomen Bereich des Systems - die Schule - ist eine anpreisende Werbefunktion des Qualitätsbegriffs bei keiner der OSB zu beobachten. Lediglich im Bereich der Erwachsenenbildung zeigt sich bei einem Drittel der von uns betrachteten Einrichtungen, dass das Qualitätsthema in einer dominant anpreisenden Form des Marketings genutzt wird. Einrichtungen der Weiterbildung verwenden den Qualitätsbegriff am häufigsten mit anpreisenden, werbeaffinen Sprachmitteln. Zugleich fällt in diesem Segment der Anteil an Nennungen mit Informationsfunktion geringer aus.

Aus der Analyse der Daten können wir die eindeutige Tendenz ablesen, dass die Thematisierung von Qualität im Sinne eines anpreisenden Marketinginstruments mit offensiven, werbeaffinen Sprachspielen ein marginales Phänomen darstellt. Das Qualitätsthema ist meist nur in eine moderate Form des Marketings eingebunden und bei der Mehrzahl der Einrichtungen ist die Verwendung des Qualitätsbegriffs mit einer deutlichen Informationsfunktion verbunden. ${ }^{15}$ Die vielfach beschworene negative Form einer Ökonomisierung der Bildung kann somit an dieser Stelle nicht belegt werden.

\subsection{Qualitätsmanagement und Qualität als Verbraucher*innenschutz?}

In keiner der 80 von uns untersuchten OSB wird der Begriff Verbraucher*innenschutz explizit verwendet. Dieser Tatbestand kann jedoch leicht zu falschen Rückschlüssen führen - denn selbstverständlich wird Verbraucher*innenschutz nicht nur thematisiert, wenn hierauf expressis verbis Bezug genommen wird.

Hinsichtlich elementarer Erwartungen an den Verbraucher*innenschutz, wie der Rechtssicherheit, kann konstatiert werden, dass sie eine zentrale Rolle für die Einrichtungen spielen. Grundlegende Präliminarien liegen vor: Als Besucher*in der Internetseite weiß man als ,gut informierter Bürger“ (Schütz 1972) mit welcher

\footnotetext{
14 Unsere Expert*innen-Interviews zeigen, dass Einrichtungen aus beiden Segmenten teils sogar auf den Marketingeffekt von QMS verzichten und in ihren OSB die von ihnen verwendeten QM-Verfahren nicht angeben.

15 In zukünftigen Forschungen könnten die mit dem Qualitätsbegriff zusammenfallenden Marketingformen und -funktionen noch differenzierter untersucht werden (vgl. etwa die Kategorisierungen der American Management Association, AMA 2021).
} 
Organisation man es zu tun hat, d.h. es werden Informationen über die Einrichtung und das dort tätige Personal gegeben. Zudem enthalten die Internetseiten Verweise auf die Allgemeinen Geschäftsbedingungen und häufig gibt es Erläuterungen zum Beschwerdemanagement.

Nun stellt sich die Frage, inwiefern die OSB QMS ausweisen und die mit den Systemen ursprünglich verbundene bildungspolitische Erwartung erfüllen und Transparenz und Orientierung liefern und damit Verbraucher*innenschutz etablieren. In den uns vorliegenden OSB nennen 36 von 60 Einrichtungen ein QMS. ${ }^{16}$ Die Unterschiede zwischen den Segmenten sind erheblich. In der Weiterbildung nennen $80 \%$ der Einrichtungen ein QMS. Dies entspricht aktuellen repräsentativen Umfragen (Ambos et al. 2018). Im Gegensatz hierzu sind es im Elementarbereich nur ca. 50\% der Einrichtungen in unserem Sample. Dies korrespondiert mit jüngsten Ergebnissen des Deutschen Kitaleitungskongresses (2018, S. 16). Andere Studien weisen jedoch deutlich höhere Werte von bis zu $78 \%$ der Einrichtungen aus (BMBF 2020, S. 100; Schreier et al. 2014, S. 159-160). Im Bereich der Sozialarbeit/Sozialpädagogik geben etwas mehr als ein Viertel der von uns betrachteten Einrichtungen ein QMS an. ${ }^{17}$

In unseren Daten zeigt sich eine hohe Diversität an QMS: Die 36 Einrichtungen, die über ein QMS verfügen, verwenden 21 verschiedene Systeme; einige Einrichtungen nutzen mehrere QMS. Im Elementarbereich werden von den zwölf Einrichtungen bzw. Trägern, die ein QMS nennen, sieben verschiedene Verfahren ausgewiesen. Im Weiterbildungsbereich nennen achtzehn Einrichtungen mit QMS insgesamt neun verschiedene Systeme. Im Bereich der Sozialpädagogik haben sechs Einrichtungen sechs verschiedene Systeme. Diese Befunde decken sich mit bestehenden Studien zu den einzelnen hier betrachteten Segmenten (Ambos et al. 2018, S. 15-16; Käpplinger und Reuter 2017, S. 15; Stiftung Warentest 2008; BMBF 2020, S. 100-101; Deutscher Kitaleitungskongress 2018, S. 16; Merchel 2005, S. 43-44; 2006, S. 199, 202-203). Mit Blick auf das pädagogisch organisierte System des lebenslangen Lernens kann man daher mit Fug und Recht von einer Inflationierung der QMS sprechen.

Zentral ist dabei der Befund, dass eine hohe Zahl segmentspezifischer QMS vorliegt. In den einzelnen Segmenten (und teilweise auch auf Träger- und Einrichtungsebene!) wird der aus der Privatwirtschaft stammende Gedanke des QM breit und diversifiziert - und damit höchst konstruktiv - angeeignet. QM wird eingemeindet, indem sehr spezifische Verfahren entwickelt werden, die der Besonderheit der Segmente, aber auch der Einrichtungen entsprechen. In der Weiterbildung können in unserem Sample sechs bereichsspezifische Systeme identifiziert werden (zwei trägereigene QMS; ,QVB` (Qualitätsentwicklung im Verbund von Bildungseinrichtungen), ,LQW`(Lernerorientierte Qualitätstestierung in der Weiterbildung); ein adaptiertes ISO-9001 QMS; ,Weiterbildung Hamburg'); im Elementarbereich sieben (,Deutsches Kindergartengütesiegel`; Evangelisches Gütesiegel BETA (Bun-

\footnotetext{
16 Hierbei wird das Schulsegment ausgespart; neben den staatlich durchgeführten Schulinspektionen finden sich kaum weitere QMS.

17 Hier ist zu berücksichtigen, dass Interviews des QUEB-Projekts zeigen, dass sozialpädagogische Einrichtungen ihre QM-Verfahren teilweise nicht in ihren OSB aufführen.
} 
desrahmenhandbuch der Bundesvereinigung Evangelischer Kindertageseinrichtungen e.V.); ,QuaSi“ (Qualität im Situationsansatz); ,IQUE‘ (Integrierte Qualitätsund Personalentwicklung); ,Paritätische Qualitätsgemeinschaft'; ,Wege zur Qualität'; Gütesiegel des KTK (Verband Katholischer Tageseinrichtungen für Kinder) und in der Sozialen Arbeit fünf (das QMS der, deQus' (Deutsche Gesellschaft für Qualitätsmanagement in der Suchttherapie e.V.); ,Paritätische Qualitätsgemeinschaft"; zwei trägereigene QMS; ein adaptiertes ISO-9001 System). Diese Befunde korrespondieren mit jüngsten Forschungsbefunden in allen hier betrachteten Segmenten (bspw. BMBF 2020, S. 100-101; Käpplinger und Reuter 2017, S. 12-14; Merchel 2005, S. 43-44; Hartz und Meisel 2007, S. 213-214).

Die hohe Zahl verschiedener QMS legt nahe, dass von einer Orientierungsfunktion jedoch nicht die Rede sein kann. Sie dürfte die Adressat*innen eher mit Desorientierung zurücklassen da es außerordentlich schwer ist, sich in der Vielzahl an Modellen zurecht zu finden und zu wissen, wofür einzelne Verfahren stehen und welcher Aussagegehalt den einzelnen Zertifizierungen zukommt. ${ }^{18}$ Gar illusorisch erscheint der Gedanke, dass sie verschiedene Modelle miteinander vergleichen könnten. ${ }^{19}$ Insofern helfen die QMS nicht oder nur in begrenztem Ausmaß dabei, eine Organisation und ein Angebot auszuwählen.

Aktuelle Rezeptionsstudien zur (Orientierungs-)Wirkung von QMS auf Adressat*innen liegen nicht vor. Zu ähnlichen Schlussfolgerungen wie wir kommen mit Blick auf die Segmente der Sozialen Arbeit und der Erwachsenenbildung jedoch auch Merchel (2006; 2004) sowie Hartz und Meisel (2007). Zudem stellen Käpplinger und Reuter resümierend für den Weiterbildungsbereich fest, dass es keine Rezeptionsstudien mit Adressat*innen gibt, die auf eine Orientierungsfunktion hinweisen (2017, S. 16). ${ }^{20}$ Für das potenzielle Publikum ist eine sachkundige Orientierung auf der Grundlage der in den OSB gelieferten Informationen im Zusammenhang mit QMS insgesamt außerordentlich schwer. ${ }^{21}$ Diese Erkenntnis wird auch dadurch gestützt, dass die in den OSB ventilierten Informationen eine Menge Vorwissen voraussetzen. Zum Teil sind sie in einer Sprache verfasst, die starke Ähnlichkeiten mit dem pädagogischen Fachjargon aufweisen.

\footnotetext{
18 Auf der Basis der OSB können wir keine Aussage über die tatsächliche Rezeption durch die Adressat*innen und die Orientierungswirkung machen. Hier besteht weiterer Forschungsbedarf.

19 Zudem werden in den OSB neben QM-Systemen auch weitere Zertifizierungen (wie bspw. AZAV) oder auch Zertifizierungsstellen neben oder anstelle der QMS genannt, ohne dass für die Leser*innen leicht erkennbar wäre, inwiefern es sich bei den Angaben um QMS handelt oder nicht.

20 Der Umstand, dass es von unserer Seite der Wissenschaft aus keineswegs unproblematisch und mühelos möglich war, einen gewissen Überblick zu gewinnen, deutet in die gleiche Richtung. Jedoch ist dabei zu berücksichtigen, dass sich die Rezeptionslogiken von Adressat*innen und Wissenschaftler*innen erheblich unterscheiden können.

21 Unsere Expert*innen-Interviews zeigen, dass Einrichtungen teilweise selber die QM-Verfahren als geringes Datum für ihre Adressat*innen wahrnehmen und auf eine Angabe in ihrer OSB verzichten.
} 


\subsection{Systemweite Homologie in der Aneignung des Qualitätsbegriffs: emphatische Klient*innenorientierung und Weiterentwicklung hoher Fachlichkeit als Verbraucher*innenschutz}

Mit Blick auf unsere Ausgangsfragen kann konstatiert werden: Die Textstellen in den OSB, die Qualität explizit thematisieren, werden nicht durch eine anpreisende, offensive Werbesprache dominiert. Allerdings dienen die QMS nicht dem Verbraucher*innenschutz im Sinne einer Orientierungsfunktion für die Adressat*innen.

Die Ergebnisse unserer qualitativen Inhaltsanalyse deuten jedoch an, dass der Aspekt des Verbraucher*innenschutzes systemweit sehr wohl aufgegriffen und systemimmanent adaptiert wurde. Das aus dem Wirtschaftssystem stammende Konzept des Verbraucher*innenschutzes wurde bereichsübergreifend über zwei Elemente angeeignet: die Stärkung der pädagogisch Anderen ${ }^{22}$ und das Selbstverständnis der Einrichtungen als lernende Organisationen - insbesondere auch die Sicherung und Weiterentwicklung hoher Fachlichkeit durch Fortbildungen und/oder die interne und externe diskursive Überprüfung und Bewertung der eigenen Arbeit (auch durch die Zielgruppen und sonstige Adressat*innen). Beide Dimensionen dominieren segmentübergreifend das Qualitätstopos. Bereits die zahlenmäßig häufige Erwähnung der pädagogisch Anderen mit dem Qualitätsbegriff verdeutlicht eine besondere Adressat*innenorientierung. Die Stärkung der Klient*innen kommt vor allem darin zum Ausdruck, dass die OSB dem Qualitätsbegriff häufig explizit folgende Aspekte zuordnen: ein dialogisches Verhältnis bzw. eine Feedbackkultur mit den Adressat*innen (einschließlich der Eltern und möglicher Auftraggeber*innen) und eine emphatische Klient*innenorientierung bei der Gestaltung des Angebots. Segmentspezifisch sind diese Aspekte durchaus unterschiedlich ausgestaltet: die emphatische Klient*innenorientierung kommt im Bereich der Schule etwa in der starken Betonung von Unterrichtsqualität zum Ausdruck (einschließlich der internen Reflexion dazu). Funktionales Äquivalent im KiTa-Bereich und der Sozialen Arbeit ist der interne und externe Austausch (im Team sowie mit Fachberater*innen, Trägern, Supervisor*innen und anderen Einrichtungen) sowie die Hervorhebung der Vorbereitung, Dokumentation und Reflexion pädagogischer Arbeit als Qualitätskriterien. Im Bereich der Erwachsenenbildung sind es Evaluationen, eine klient*innenorientierte Angebotsgestaltung sowie die Auswahl, die Qualifikationen und die Kontrolle der teils unabhängigen Dozent*innen.

Mittels der in den OSB inflationär vorkommenden Beschreibungen, wie „Wir [als Organisation] sind lernfähig“ und „Wir entwickeln uns ständig weiter“, legen die Einrichtungen zudem einen Fokus auf kontinuierliche Weiterentwicklung. ${ }^{23}$ Segmentübergreifend werden die organisationale Weiterentwicklung und die indi-

\footnotetext{
22 Der Begriff der pädagogisch Anderen wird als Sammelbegriff für die Adressat*innen pädagogischer Organisationen verwendet (Nittel 2017, S. 141ff.).

23 Nachfolgend werden weitere Textpassagen angeführt, die die Weiterentwicklung bzw. das organisationale Lernen betonen: „Durch interne und externe Fachberatung ermöglichen wir die konzeptionelle und praktische Weiterentwicklung.“; ,Intern werden geltende Qualitätsstandards im regulären Betrieb (Teambesprechungen, Entwicklungsgespräche, Weiterentwicklung der Arbeit) überprüft und weiterentwickelt. Die externen Qualitätsprüfungen wurden im Team ausgewertet, die Ergebnisse wurden zur Veränderungsplanung innerhalb unserer Qualitätsentwicklung mit herangezogen.“.
} 
viduelle Fortbildung des Personals ins Zentrum der Aufmerksamkeit gerückt. Bemerkenswert ist, dass das aus dem Wirtschaftssystem stammende Konzept der Qualitätssicherung und die Formulierung von Prozessstandards dagegen weniger bedeutsam sind. Die OSB nehmen in allen Segmenten zwar Bezug auf Standards und den Aspekt der Sicherung. Legt man jedoch reine Häufigkeitsauszählungen zugrunde, zeigt sich, dass die Bezüge zur Weiterentwicklung der Einrichtungen und ihrer Praxis in allen Segmenten sehr deutlich überwiegen. Eine bloße Übernahme ökonomischer Konzepte ist insofern nicht erfolgt. Vielmehr wird deutlich, dass der aus dem Wirtschaftssystem stammende Qualitätstopos mit dem systemeigenen Diskurs des lebenslangen Lernens verbunden wurde. Die Einrichtungen applizieren die Idee der Qualität im Sinne lebenslangen Lernens als reflexiven Mechanismus auf sich selbst (vgl. Nittel und Kilinc 2020; Spahn et al. 2019). Der Qualitätsdiskurs und das lebenslange Lernen bilden hier eine systemweite Einheit. ${ }^{24}$

Mit Blick auf das System lässt sich somit festhalten, dass aus dem wirtschaftsnahen Konzept des Verbraucher*innenschutzes die emphatische Adressat*innenorientierung bzw. die permanente reflexive und diskursive Überprüfung des eigenen Handelns (auch und gerade durch die Bewertung von Seiten der Zielgruppen) sowie Fortbildungen und darauf aufbauende Qualitätsentwicklung geworden sind. Verbraucher*innenschutz - so könnte man schlussfolgern - wurde transformiert und ist insofern in den OSB verankert - jedoch nicht im ursprünglichen Sinne der Orientierungsfunktion.

\subsection{Die Legitimationsfunktion des Qualitätstopos}

Die Adaptierung des Verbraucher*innenschutzes verweist auf eine übergeordnete, segmentübergreifende Funktion des Qualitätsbegriffs in den OSB: eine Legitimationsfunktion. QMS und deren Zertifizierungen ebenso wie die im Wirtschaftssystem damit verbundenen Konzepte (wie der Verbraucher*innenschutz) werden von den Organisationen als normative Erwartung erlebt. Die Thematisierung von QMS in den OSB stellt insofern eine Legitimationsform gegenüber der Umwelt dar (Käpplinger und Reuter 2017; Merchel 2004, S. 58). Mit den in der Inhaltsanalyse identifizierten Qualitätsdimensionen weisen die Einrichtungen zudem nach, dass sie zentrale Grundideen des Qualitätstopos aus dem Wirtschaftssystem aufgegriffen haben. Indem sie in ihren OSB hervorheben, dass sie sich - auch von ihren Ziel- und Adressat*innengruppen - intern und extern überprüfen und bewerten lassen, weisen sie sich als Organisationen aus, die den Aspekt des Verbraucher*innenschutzes aufgenommen haben. Mit den Verfahren und ihren Ergebnissen legitimieren sie sich „nach innen und nach außen“ (Merchel 2005, S. 46). Auch die Betonung, die pädagogische Arbeit und das Angebot an den pädagogisch Anderen auszurichten, kommuniziert, dass sie den aus dem Wirtschaftssystem stammenden Aspekt der Kund*innenorientierung systemintern transformieren und umsetzen. Das Sprechen

\footnotetext{
${ }^{24}$ Das lebenslange Lernen der einzelnen Gesellschaftsmitglieder und der Primat des fortwährenden organisationalen Lernens sind durch den von George Herbert Mead adaptierten Begriff des Universalisierungsmechanismus miteinander verbunden (vgl. die LOEB-Studie, in der das lebenslange Lernen als Universalisierungsmechanismus identifiziert wurde; Nittel und Tippelt 2019, S. 31-35).
} 
über Qualität hat auf der Systemebene somit die Funktion, die performative Basis für die Inszenierung eines ambitionierten Leistungsethos gegenüber den Adressat*innen zu schaffen. Das darf und muss auch als Reaktion darauf verstanden werden, dass dem System des lebenslangen Lernens in den 1990er-Jahren mit der Forderung nach Qualität Vertrauen entzogen wurde (Klieme und Tippelt 2008). Die Entstehung des Qualitätsdiskurses war segmentübergreifend eng verwoben mit der Forderung nach verbesserter Legitimation (zum Hochschulbereich exemplarisch Wolter und Kerst 2008, S. 138).

\section{Weiterführende Betrachtungen und praktische Schlussfolgerungen: Die bildungs-bereichsübergreifende Sozialisation der Adressat*innen auf den Qualitätsbegriff und die Konstitution eines systemweiten Relevanzsystems guter pädagogischer Praxis}

Die Struktur des pädagogisch organisierten Systems des lebenslangen Lernens und die OSB weisen eine Homologie auf: Die enorme - empirisch nachgewiesene Orientierungskraft des Qualitätsgedankens und die faktische Allgegenwart organisationaler Qualitätsentwicklung korrespondieren mit der Ubiquität von Verweisen auf Qualität in den OSB. Implizite Thematisierungen finden sich in jeder der von uns betrachteten OSB. Zudem wird der Qualitätsbegriff in den OSB im Durchschnitt fünf Mal explizit erwähnt und die Einrichtungen benennen dabei zahlreiche Qualitätsdimensionen. Der Qualitätsbegriff erweist sich somit als omnipräsent in den OSB. Das hat zwar auch mit den formalen Merkmalen dieser Textgattung zu tun - schließlich hat keine Organisation ein Interesse daran, sich schlecht zu präsentieren. Dennoch halten wir diesen auf den ersten Blick profan wirkenden Befund für bemerkenswert. Insbesondere deshalb, weil der Qualitätstopos somit ein systemweites Phänomen ist, das sowohl - wie oben gezeigt - auf zentralen inhaltlichen Ebenen (der emphatischen Klient*innen-Orientierung und der Fokussierung auf Weiterentwicklung von Fachlichkeit), aber auch in quantitativer Hinsicht von allen Segmenten in tendenziell gleicher Weise in den OSB aufgegriffen wird. Auffällig ist der Befund, dass der Qualitätsbegriff in OSB systemweit in quantitativer Hinsicht in sehr ähnlicher Weise aufgegriffen wird. ${ }^{25}$ Unterschiede in der Häufigkeit des Verweises auf den Begriff sind kein Unterscheidungsmerkmal zwischen den Segmenten - sondern eher zwischen Organisationen.

Indem Organisationen der Elementar- und Schulpädagogik, der Sozialarbeit/ Sozialpädagogik und Erwachsenenbildung simultan auf Qualität rekurrieren, konstituieren und reproduzieren sie ein bildungsbereichsübergreifendes Relevanzsystem. ${ }^{26}$ Sie sensibilisieren, sozialisieren und konditionieren die pädagogisch Anderen und deren gesetzliche Vertreter*innen auf Aspekte, die bedeutsam sind und beachtet werden sollten. Die Organisationen schaffen dadurch einen Resonanzboden, be-

\footnotetext{
25 Lediglich im Bereich der Sozialen Arbeit wird der Qualitätsbegriff mit im Durchschnitt 3,5 Nennungen in den OSB etwas seltener genannt. Auch hier besteht jedoch hohe Heterogenität.

26 Die Konditionierung auf den Qualitätsbegriff kommt auch darin zum Ausdruck, dass mögliche äquivalente Begriffe oder Synonyme, wie ,Güte‘ oder ,qualitativ‘, so gut wie nie in den OSB vorkommen.
} 
stimmen den kollektiven Aufmerksamkeitsfokus und lenken die Adressat*innen. Sie erklären auf subtile Weise, was wichtig bzw. unwichtig ist und etablieren zugleich ein kollektiv geteiltes Anspruchsniveau und diesbezügliche Erwartungen. Wie in einem hidden curriculum sorgen die Organisationen dafür, dass dadurch eine systemweite Definition guter pädagogischer Praxis entsteht.

Auf grundlagentheoretischer Ebene verdeutlichen die Homologien in der Adaptierung des Qualitätstopos (und die damit einhergehende Definition guter pädagogischer Praxis) ebenso wie die Überschneidungen des Qualitätsthemas mit der Orientierungsidee des lebenslangen Lernens die Systembildungstendenzen - hin zu einem pädagogisch organisierten System des lebenslangen Lernens (Nittel 2017). Zugleich wird deutlich, dass von einer bloßen Ökonomisierung des Systems durch die Adaptierung des Qualitätskonzepts aus dem Wirtschaftssystem nicht die Rede sein kann. Die vorliegenden Daten zeigen vielmehr die konstruktiven Aushandlungsund Aneignungsprozesse, die das System mit Blick auf das Qualitätsphänomen vollzieht.

Unseren Befunden kommt zudem Praxisrelevanz in mehreren Punkten zu: Aus unserer Sicht kann es für pädagogische Organisationen gewinnbringend sein, an die Idee des Verbraucher*innenschutzes wieder anzuknüpfen. Es handelt sich dabei um einen positiven Aspekt von Ökonomisierung, der nicht versteckt werden sollte. Die Adaptionsleistung der Einrichtungen und die dem Verbraucher*innenschutz dienenden Elemente der OSB sollten selbstbewusst herausgestellt werden. Sie könnten das öffentliche Bild und die Anerkennung der Organisationen und der Profession stärken. Mit positivem Selbstverständnis kann die Praxis zudem hervorheben, dass sich ihre OSB abheben von einer vermeintlich rein anpreisenden Werbesprache. Die Inflationierung des Qualitätsbegriffs in den OSB der Organisationen bedeutet nicht, wie wir zeigen konnten, dass der Begriff zu einem rein anpreisenden Marketinginstrument geworden ist. Vertreter*innen der Einrichtungen können die hier dargelegten Befunde als Anlass interpretieren, mit einem positiven Stolz auf die Stilelemente der OSB zu blicken und einzugehen. Als praktische Schlussfolgerung ergibt sich aus den Erfahrungen im QUEB-Projekt zudem, dass Einrichtungen die Textsorte der OSB im Internet in ihrer Bedeutung noch ernster nehmen sollten. Einzelne Einrichtungen sehen darin lediglich ein notwendiges Übel. Sie sind jedoch für die Einrichtungen objektiv relevant: Forschungsergebnisse zeigen die Relevanz von Konzeptionen und Profilen für Bildungsentscheidungen (Schwarz et al. 2018) und Adressat*innen holen über die online verfügbaren OSB entsprechende Informationen ein. Zudem verweist der Artikel - zumindest implizit - auf eine systemische Relevanz der OSB: Vergleicht man heutige OSB im Internet mit den 2014 im Rahmen des LOEB-Projekts gesammelten Daten, so wird deutlich, wie schnell sich Digitalität als kulturelle Praktik der Organisationsführung und der Kommunikation mit der Umwelt im System des lebenslangen Lernens verbreitet und dass organisationales Lernen im Bereich der Digitalisierung in beachtlicher Breite stattgefunden hat. Darauf können die pädagogischen Organisationen mit Stolz blicken. Die oft zu findende Defizithypothese mit Bezug auf das Thema Digitalisierung kann vor dem Hintergrund der Entwicklung der OSB nicht bestätigt werden.

Funding Open Access funding enabled and organized by Projekt DEAL. 
Open Access Dieser Artikel wird unter der Creative Commons Namensnennung 4.0 International Lizenz veröffentlicht, welche die Nutzung, Vervielfältigung, Bearbeitung, Verbreitung und Wiedergabe in jeglichem Medium und Format erlaubt, sofern Sie den/die ursprünglichen Autor(en) und die Quelle ordnungsgemäß nennen, einen Link zur Creative Commons Lizenz beifügen und angeben, ob Änderungen vorgenommen wurden.

Die in diesem Artikel enthaltenen Bilder und sonstiges Drittmaterial unterliegen ebenfalls der genannten Creative Commons Lizenz, sofern sich aus der Abbildungslegende nichts anderes ergibt. Sofern das betreffende Material nicht unter der genannten Creative Commons Lizenz steht und die betreffende Handlung nicht nach gesetzlichen Vorschriften erlaubt ist, ist für die oben aufgeführten Weiterverwendungen des Materials die Einwilligung des jeweiligen Rechteinhabers einzuholen.

Weitere Details zur Lizenz entnehmen Sie bitte der Lizenzinformation auf http://creativecommons.org/ licenses/by/4.0/deed.de.

\section{Literatur}

Ambos, I., Koscheck, S., Martin, A., \& Reuter, M. (2018). Qualitätsmanagementsysteme in der Weiterbildung: Ergebnisse wbmonitor Umfrage 2017. https://wbmonitor.bibb.de/index.php?cmd= usrInquiryResults\&inq_id=89. Zugegriffen: 20. Jan. 2021.

American Marketing Association (2021). https://www.ama.org/the-definition-of-marketing-what-ismarketing/. Zugegriffen: 1. Sept. 2021.

Bessler, H. (1972). Aussagenanalyse. Düsseldorf: Bertelsmann.

BMBF (2020). Gute-KiTa-Bericht 2020. https://www.bmfsfj.de/bmfsfj/service/publikationen/gute-kitabericht-2020-163402. Zugegriffen: 21. Febr. 2021.

Brückers, W. (1998). Qualität in der Sozialen Arbeit: 10 Bausteine einer AWO-Strategie „Qualitätsmanagement". Theorie und Praxis der Sozialen Arbeit, 11, 402-409.

Dellori, C. (2016). Die absolute Metapher, lebenslanges Lernen': Eine Argumentationsanalyse. Wiesbaden: Springer.

Deutsche Gesellschaft zur Zertifizierung von Managementsystemen Zur Historie von ISO 9001: Eine Erfolgsgeschichte. https://www.dqs.de/blog/qualitaet/historie-iso-9001/. Zugegriffen: 3. März 2021.

Deutscher Kitaleitungskongress (2018). Befragung zur Wertschätzung und Anerkennung von Kita-Leitungen. Die Kita-Leitung im Zentrum der Qualitätsdebatte. Wolters Klouwer. https://www.deutscherkitaleitungskongress.de/assets/documents/placeholder/DKLK-Studie_2018.pdf. Zugegriffen: 21. Febr. 2021.

Dittrich, G. (o.J.). Datenbank ProKiTa: Praxisforschung und Modellprojekte in den Bereichen Kindertagesstätten und Tagespflege. Deutsches Jugendinstitut. https://www.dji.de/index.php?id=40994. Zugegriffen: 14. Feb. 2021.

Goffman, E. (1963). Stigma. Englewood Cliffs: Prentice Hall.

Goffman, E. (1967). Interaction ritual. Garden City: Anchor Books.

Grönroos, C. (2006). On defining marketing: finding a new roadmap for marketing. Marketing Theory, 6(4), 395-417.

Hartz, S., \& Meisel, K. (2007). Qualitätsmanagement. Bielefeld: wbv.

Hartz, S., \& Meisel, K. (2011). Qualitätsmanagement. Bielefeld: wbv.

Helmke, A., Hornstein, W., \& Terhart, E. (Hrsg.). (2000). Qualität und Qualitätsmanagement im Bildungsbereich: Schule, Sozialpädagogik, Hochschule. Weinheim: Beltz.

International Organization for Standardization (ISO) (o.J.). https://www.iso.org/home.html. Zugegriffen: 19. Dez. 2021

Kallmeyer, W., \& Schütze, F. (1977). Zur Konstitution von Kommunikationsschemata der Sachverhaltsdarstellung. In D. Wegner (Hrsg.), Gesprächsanalysen (S. 159-274). Hamburg: Buske.

Käpplinger, B., \& Reuter, M. (2017). Qualitätsmanagement in der Weiterbildung: Entstehung und Entwicklung. WISO Diskurs, 15, 10-15.

Klieme, E., \& Tippelt, R. (Hrsg.). (2008). Qualitätssicherung im Bildungswesen: Eine aktuelle Zwischenbilanz. Weinheim: Beltz.

Kuckartz, U. (2018). Qualitative Inhaltsanalyse. Weinheim: Beltz.

Kuper, H., Thiel, F. (2018). Erziehungswissenschaftliche Institutionen- und Organisationsforschung. In R. Tippelt, B. Schmidt-Hertha (Hrsg.), Handbuch Bildungsforschung, 4. Auflage (S. 587-606).

Mayring, P. (2015). Qualitative Inhaltsanalyse. Grundlagen und Techniken. Weinheim: Beltz. 
Merchel, J. (2004). Zertifizierung und Qualitätssiegel: Risiken für den Prozess der Qualitätsentwicklung in der Sozialen Arbeit. In F. Peterander \& O. Speck (Hrsg.), Qualitätsmanagement in sozialen Einrichtungen (S. 44-63). München: Ernst Reinhardt.

Merchel, J. (2005). Was hat die Qualitätsdebatte in der Jugendhilfe gebracht? Versuch einer Zwischenbilanz. Archiv für Wissenschaft und Praxis der sozialen Arbeit, 36, 38-59.

Merchel, J. (2006). Qualitätsmanagement in der Sozialen Arbeit: Erfahrungen zur Verarbeitung und zur Umsetzung des Themas „Qualität“. Der pädagogische Blick, 14(4), 195-208.

Merchel, J. (2013). Qualitätsmanagement in der Sozialen Arbeit: Eine Einführung (4. Aufl.). Weinheim: Beltz Juventa.

Nittel, D. (2017). Ein System in statu nascendi: Auf dem Weg zum pädagogisch organisierten System des lebenslangen Lernens? In R. Arnold \& W. Neuser (Hrsg.), Beobachtung des Wissens: Das Wissen des Beobachters (S. 119-166). Baltmannsweiler: Schneider Hohengehren.

Nittel, D., \& Kilinc, M. (2020). Die Qualität der Qualität? Empirische Annäherung an einen reflexiven Mechanismus im pädagogisch organisierten System des lebenslangen Lernens. Zeitschrift für Qualitative Forschung, 21(2), 273-290.

Nittel, D., \& Tippelt, R. (2018). Die Resonanz des lebenslangen Lernens im Erziehungs- und Bildungswesen: Eine vergleichende Untersuchung beruflicher und institutioneller Selbstbeschreibungen. Düsseldorf: Edition Hans Böckler. unter Mitarbeit von B. Lindemann, P. Kettner, J. Schütz \& J. Wahl

Nittel, D., \& Tippelt, R. (2019). Pädagogische Organisationen im System des lebenslangen Lernens. Bielefeld: wbv.

Nittel, D., Schütz, J., \& Tippelt, R. (2014). Pädagogische Arbeit im System des lebenslangen Lernens: Ergebnisse komparativer Berufsgruppenforschung. Weinheim, Basel: Beltz Juventa.

Schreier, I., Krause, M., Brandl, M., \& Nicko, O. (2014). AQUA. Arbeitsplatz und Qualität in Kitas. http:// www.aqua-studie.de/Dokumente/AQUA_Endbericht.pdf. Zugegriffen: 21. Febr. 2021.

Schütz, A. (1972). Der gut informierte Bürger. In A. Brodersen (Hrsg.), Alfred Schütz: Gesammelte Aufsätze (Bd. 2, S. 85-101). Den Haag: Nijhoff.

Schütze, F. (1978). Strategische Interaktion im Verwaltungsgericht: Eine soziolinguistische Analyse zum Kommunikationsverlauf im Verfahren zur Anerkennung als Wehrdienstverweigerer. In W. Hassemer, W. Hoffmann-Riem \& M. Weiss (Hrsg.), Schriften der Vereinigung für Rechtssoziologie (Bd. 2, S. 19-100). Baden-Baden: Nomos.

Schwarz, J., Habeck, C., Gruehn, S., \& Koinzer, T. (2018). „Welche Schule passt zu meinem Kind?“. Elterliche Schulwahlentscheidungen und die Frage der Passung im öffentlichen und privaten Grundschulwesen. Zeitschrift für Pädagogik, 64(5), 612-634.

Spahn, C., Nittel, D., Hodapp, B., \& Kilinc, M. (2019). Qualitätsentwicklungen im pädagogisch organisierten System des lebenslangen Lernens. Zeitschrift für Bildungsverwaltung, 35(2), 5-21.

Spieß, C. K., \& Tietze, W. (2002). Qualitätssicherung in Kindertageseinrichtungen: Gründe, Anforderungen und Umsetzungsüberlegungen für ein Gütesiegel. Zeitschrift für Erziehungswissenschaft, 1, 139-162.

Stiftung Warentest (2008). Transparenz ist nicht in Sicht. https://www.test.de/QualitaetsmanagementTransparenz-ist-nicht-in-Sicht-1531451-0/. Zugegriffen: 10. März 2021.

Tietze, W. (2008). Qualitätssicherung im Elementarbereich. In E. Klieme \& R. Tippelt (Hrsg.), Qualitätssicherung im Bildungswesen: Eine aktuelle Zwischenbilanz (S. 16-35). Weinheim: Beltz.

Wahl, J., Nittel, D., \& Tippelt, R. (2022). Pädagogische Organisationen: Die Verschränkung von Träger, Einrichtung und Gemeinschaft. Zeitschrift für Pädagogik, 68(3).

Weiterbildung Hessen e. V. (Hrsg.). Unsere Ziele. https://weiterbildunghessen.de/ueber-uns. Zugegriffen: 3. März 2021.

Wolter, A., \& Kerst, C. (2008). Akkreditierung als Verfahren der Qualitätssicherung von Studiengängen in Deutschland. In E. Klieme \& R. Tippelt (Hrsg.), Qualitätssicherung im Bildungswesen: Eine aktuelle Zwischenbilanz (S. 135-155). Weinheim: Beltz.

Wuppertaler Kreis e.V. (Hrsg.). (1996). Qualitätsmanagement in der Weiterbildung nach DIN EN ISO 9000ff:: Ein Leitfaden. Köln: Deutscher Wirtschaftsdienst. 